\title{
Concentration dependence of diffusion coefficient at various molecular weights and temperatures
}

\author{
Charles C. Han \\ Center for Materials Research, Polymer Science and Standards Division, National Bureau \\ of Standards, Washington, D.C. 20234, USA
}

\author{
and A. Ziya Akcasu \\ Department of Nuclear Engineering, The University of Michigan, Ann Arbor, Michigan, \\ 48109, USA
}

(Received 30 December 1980)

\begin{abstract}
The effect of temperature on the concentration dependence of the diffusion coefficient $D(c)$ is investigated experimentally by light scattering using polystyrene with $M_{w}=179000$ and 900000 in toluene and cyclohexane at several temperatures. It is found that $D(c)$ decreases with concentration under the theta condition, and increases in good solvents. The continuous transition from theta $(\theta)$ to good solvent behaviour is explored, and the results are compared with the existing theoretical predictions.
\end{abstract}

\section{INTRODUCTION}

In this paper we present an experimental study of the concentration dependence of the diffusion coefficient of polymers in solution as a function of molecular weight and temperature. We restricted ourselves to dilute solutions and small scattering wave vectors $q$. Experimental data will be presented and compared with theoretical predictions.

In equilibrium measurements, the static light scattering yields information on the second virial coefficient when $q \rightarrow 0$. The structure factor $S(q)$ gives the radius of gyration of the polymer molecules as the concentration approaches zero. At the theta condition, $S(q \rightarrow 0)$ is independent of concentration to the first order because the second virial coefficient, $A_{2}$, vanishes by definition, and the structure factor gives the 'unperturbed' dimension or radius of gyration of polymer molecules ${ }^{1}$.

In dynamic measurements, the intermediate scattering function $S(q, t)$ yields the diffusion coefficient, $D$, in the small $q$ limit. By using the Gibbs-Duhem expression ${ }^{1}$, the diffusion coefficient can be written as:

$$
D=\frac{k_{B} T}{f}\left(1-\frac{N_{A} v_{1}}{M} c\right)\left(1+2 A_{2} M c+\ldots\right)
$$

where $k_{B} T$ is the temperature of the solution, $f$ is the frictional coefficient of the polymer molecule in solution. $A_{2}$ is the second virial coefficient, $v_{1}$ is the partial specific volume of polymer with molecular weight $M$, and $N_{A}$ is the Avogadro Number. In this expression, the concentration dependence of $D$ is separated into two parts:
The first contribution is from the chemical potential which involves the virial coefficients. The second contribution is due to the hydrodynamic interaction which is included in the frictional coefficient $f$. One can immediately draw several conclusions: (1) The concentration dependence of $D$ can be non-zcro at $\theta$-temperature, because $f$ may still be concentration dependent even at $T$ $=0,(2)$. The sign and magnitude of the initial slope $S$ of $D$ as a function of concentration at a given temperature and molecular weight will depend on the relative magnitudes of $A_{2}$ and the concentration coefficient of $f$. It has been observed that $S$ is negative and molecular weight independent at the 0 -temperature ${ }^{2.3}$, whereas it is positive and slightly molecular-weight dependent in good solvents. It is the calculation of $f^{4.5 .6}$ which depends on the model and approximations used.

The purpose of this paper is to investigate the temperature and molecular weight dependence of $S$ by measuring the diffusion coefficient by dynamic light scattering, and compare the results with various theoretical models reported in the literature. Particular attention is paid to the continuous transition from the theta to good solvent behaviour of the diffusion coefficient.

\section{EXPERIMENTAL}

Sample preparation

NBS SRM-705 polystyrene with $M_{w}=179000$ and Pressure Chemical 61208 polystyrene with $M_{w^{\prime}}=900000$ were used in this study. Both samples have narrow molecular weight distributions with $M_{w} / M_{n} \sim 1.06$. ACS 
Table 1 Diffusion coefficient at infinite dilution, $D(0)$, and slope, $S$, for different molecular weights

\begin{tabular}{|c|c|c|c|c|}
\hline$M_{W} \times 10^{-3}$ & Solvent & $T\left({ }^{\circ} \mathrm{C}\right)$ & $D(0) \times 10^{7}\left(\mathrm{~cm}^{2} \mathrm{~s}^{-1}\right)$ & $S \times 10^{7}\left(\mathrm{~cm}^{5} \mathrm{sec}^{-1} \mathrm{gm}^{-1}\right)$ \\
\hline 179 & Cyclohexane & $\begin{array}{l}35 \\
38 \\
40.4 \\
45 \\
55\end{array}$ & $\begin{array}{l}3.23 \pm 0.02 \\
3.37 \pm 0.05 \\
3.64 \pm 0.01 \\
3.83 \pm 0.04 \\
4.61 \pm 0.08\end{array}$ & $\begin{array}{rr}-82.0 \pm & 3.8 \\
-42.6 \pm & 8.5 \\
-69.0 \pm & 2.0 \\
-35.0 \pm & 7.5 \\
4.2 \pm & 18.3\end{array}$ \\
\hline 900 & Cyclohexane & $\begin{array}{l}45 \\
55\end{array}$ & $\begin{array}{l}1.49 \pm 0.02 \\
1.83 \pm 0.03\end{array}$ & $\begin{array}{rr}-7.4 \pm & 4.3 \\
11.2 \pm & 8.7\end{array}$ \\
\hline 179 & Toluene & $\begin{array}{l}23 \\
55\end{array}$ & $\begin{array}{l}3.59 \pm 0.25 \\
5.09 \pm 0.03\end{array}$ & $\begin{array}{r}85.9 \pm 73.7 \\
198.1 \pm 10.1\end{array}$ \\
\hline
\end{tabular}

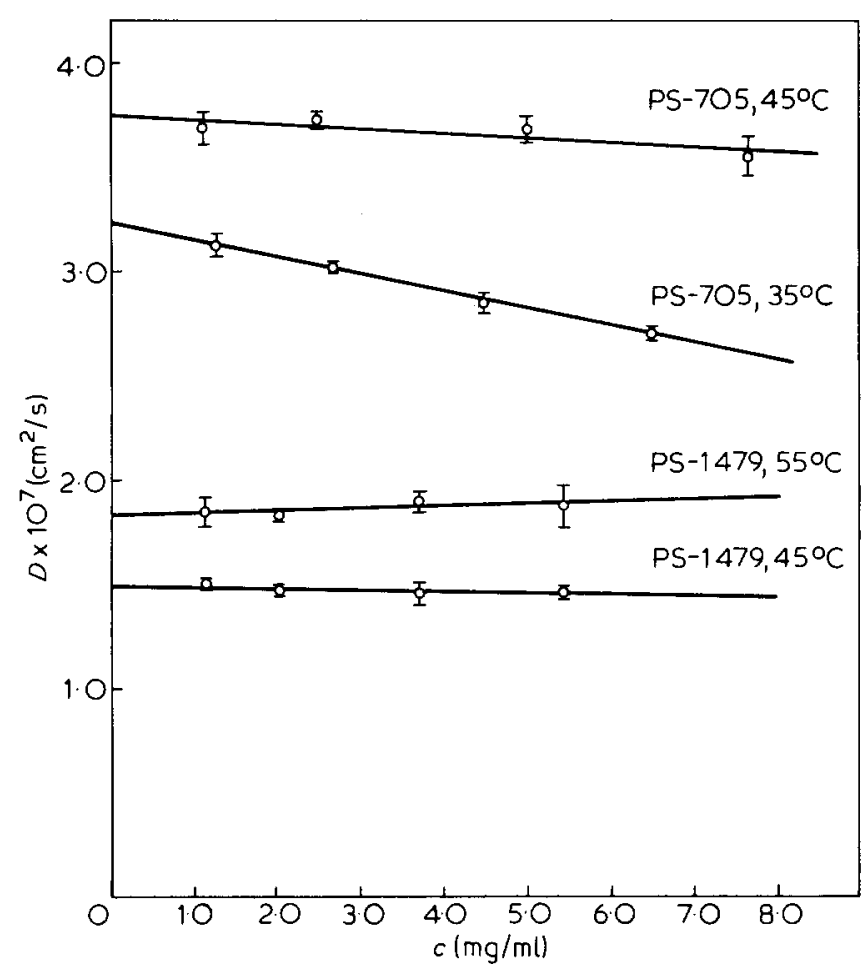

Figure 1 Some of the diffusion coefficients, $D(c)$, as a function of concentration

spectro grade cyclohexane and toluene were used for preparing the solutions. Cyclohexane solutions were kept at $40^{\circ} \mathrm{C}$, stirred gently overnight, before being used for the scattering experiment. All solutions were filtered through an $0.45 \mu \mathrm{m}$ filter into scattering cells directly.

\section{Quasielastic light scattering}

All scattering experiments were carried out on a photon counting spectrometer ${ }^{7}$. A $4880 \AA$ line from an Ar-ion laser was used for this experiment. Cylindrical sample cells were used and mounted at the centre of a temperature controlled, refractive index matched bathgoniometer. Temperatures were controlled in the experiment to within $0.2^{\circ} \mathrm{C}$ about specified values.

The intermediate scattering function $S(q, t)$ is obtained from the experimental time correlation function, $C(q, t)$, of the scattering light as ${ }^{8}$

$$
C(q, t)=1+\beta S(q, t)^{2}
$$

where $\beta$ is treated as a parameter.

Here, we have restricted our attention to small $q$-values for which $q R_{g} \ll 1$ where $R_{g}$ is the radius of gyration of a single polymer chain. In this $q$-region $S(q, t)$ can be represented by a single exponential $\exp [-\Gamma t]$. Polystyrene solutions of a given solvent, temperature, molecular weight and concentration were studied at several different angles. The exponent $\Gamma$ was extracted in each case by fitting an exponential to the measured $S(q, t)$. Then, $\Gamma$ at different angles is fitted to $D q^{2}$ to obtain the diffusion coefficient for a particular molecular weight in a given solvent as function of temperature and concentration.

The diffusion coefficients for a particular molecular weight and temperature with different concentrations were then fitted to a linear concentration dependence according to

$$
D(c)=D(0)+S c
$$

Results of diffusion coefficient at infinite dilution, $D(0)$, and the slope $S$ were collected in Table $l$ for different molecular weights, temperatures and solvents. Some of the diffusion coefficients of polystyrene in cyclohexane are shown in Figure 1, where the change of slope from negative to positive as a function of temperature is demonstrated for both samples.

For the convenience of comparison of theories, the diffusion coefficient is expressed as

$$
D(c)=D(0)\left(1+k_{D} c_{r}\right)
$$

with concentration coefficient $k_{D}$, and concentration $c_{v}$ measured in the volume fraction. The slope of concentration dependence from equation 3 is converted to $k_{D}$ by using

$$
c_{r}=c N_{A} v_{h} / M
$$

where $v_{h}$ is the hydrodynamic volume of a polymer molecule. This leads to

$$
k_{D} \frac{N_{A} l_{h}}{M}=\frac{S}{D(0)}
$$

Using $v_{h}=(4 \pi / 3) R_{H}{ }^{3}$ where $R_{H}$ is the hydrodynamic radius defined by $R_{H}=k_{B} T / 6 \pi \mathrm{D}(0)$, one finds

$$
k_{D}=\frac{S M}{D(0) N_{A}(4 \pi / 3)\left[k_{B} T / 6 \pi \eta D(0)\right]^{3}}
$$

The concentration coefficient, $k_{D}$, obtained from equation 7 is collected in Table 2, together with the solvent viscosity, 
Table 2 Parameters for polystyrenes in various solvents

\begin{tabular}{|c|c|c|c|c|c|c|}
\hline$M_{W} \times 10^{-3}$ & Solvent & $T\left({ }^{\circ} \mathrm{C}\right)$ & $\eta(c . p)$. & $A_{2}\left(\mathrm{~mol} \mathrm{~cm} / \mathrm{gm}^{2}\right)^{a}$ & $x$ & $k_{D}$ \\
\hline 179 & Cyclohexane & $\begin{array}{l}35 \\
38 \\
40.4 \\
45 \\
55\end{array}$ & $\begin{array}{l}0.73 \\
0.70 \\
0.67 \\
0.62 \\
0.52\end{array}$ & $\begin{array}{l}0 \\
1.95 \times 10^{-5} \\
3.5 \times 10^{-5} \\
6.0 \times 10^{-5} \\
11.0 \times 10^{-5}\end{array}$ & $\begin{array}{l}0 \\
0.41 \\
0.51 \\
0.59 \\
0.70\end{array}$ & $\begin{array}{r}-2.34 \pm 0.16 \\
-1.00 \pm 0.20 \\
-1.62 \pm 0.50 \\
-0.69 \pm 0.15 \\
0.06 \pm 0.26\end{array}$ \\
\hline 900 & Cyclohexane & $\begin{array}{l}44 \\
55\end{array}$ & $\begin{array}{l}0.62 \\
0.52\end{array}$ & $\begin{array}{r}6.0 \times 10^{-5} \\
11.0 \times 10^{-5}\end{array}$ & $\begin{array}{l}0.67 \\
0.82\end{array}$ & $\begin{array}{r}-0.11 \pm 0.07 \\
0.14 \pm 0.12\end{array}$ \\
\hline 179 & Toluene & $\begin{array}{l}23 \\
55\end{array}$ & $\begin{array}{l}0.55 \\
0.40\end{array}$ & $\begin{array}{l}3.5 \times 10^{-4} \\
3.5 \times 10^{-4}\end{array}$ & $\begin{array}{l}0.94 \\
0.88\end{array}$ & $\begin{array}{l}1.28 \pm 1.09 \\
1.68 \pm 0.09\end{array}$ \\
\hline $\begin{array}{c}1800 \\
860 \\
411 \\
160 \\
97.2 \\
51.0 \\
20.4\end{array}$ & $\mathrm{THT}^{-} \boldsymbol{b}$ & $\begin{array}{l}25 \\
25 \\
25 \\
25 \\
25 \\
25 \\
25\end{array}$ & & & $\begin{array}{l}1.02 \\
1.03 \\
0.97 \\
0.96 \\
0.99 \\
0.95 \\
0.93\end{array}$ & $\begin{array}{l}1.2 \\
1.15 \\
1.19 \\
1.09 \\
1.06 \\
0.89 \\
0.71\end{array}$ \\
\hline $\begin{array}{c}26.8 \\
89 \\
140 \\
314 \\
661 \\
887\end{array}$ & Toluene & $\begin{array}{l}25 \\
25 \\
25 \\
25 \\
25 \\
25\end{array}$ & & & $\begin{array}{l}0.97 \\
1.11 \\
1.12 \\
1.04 \\
1.03 \\
1.14\end{array}$ & $\begin{array}{l}0.91 \\
1.64 \\
1.79 \\
1.84 \\
2.06 \\
2.34\end{array}$ \\
\hline
\end{tabular}

a Second virial coefficients for PS in toluene are from Ref 21 for PS in cyclohexane at $45^{\circ} \mathrm{C}$ and $55^{\circ} \mathrm{C}$ are from Ref 22 for $38^{\circ} \mathrm{C}$ and $40.4^{\circ} \mathrm{C}$ are interpolated from Ref 22

$b$ All data of PS in THF used are from Ref 8

$\eta$, and second virial coefficient, $A_{2}$. The parameter $X$. which is the ratio of corresponding hard sphere radius. $\bar{S}$, to the hydrodynamic radius $R_{H}$, is also calculated by using $^{11}$

$$
A_{2}=16 \pi N_{4} \bar{S}^{3} / 3 M^{2}
$$

and shown in Table 2.

The experimental results of this study as well as the results by Mandema-Zeldenrust ${ }^{9}$ and Selser $^{10}$ are compared with the theoretical predictions in Figure 2.

\section{DISCUSSIONS AND CONCLUSIONS}

The theoretical curves in Figure 2 are reproduced from the accompanying paper $^{11}$. In the good solvent limit, corresponding to the larger values of $X=\bar{S} / R_{H}$, the data is better represented by the theoretical predictions $k_{D}=3.2\left(\bar{S} / R_{H}\right)^{3}-1$ by Yamakawa $(\mathrm{Y})^{4}$, and $k_{D}$ $=\left(\bar{S} / R_{H}\right)^{2}\left[8\left(\bar{S} / R_{H}\right)-6\right]$ by Akcasu and Benmouna $(\mathrm{AB})^{12}$. The latter was obtained by replacing the distance between two monomers belonging to two different chains by the distance of their centre of masses. As pointed out by (AB) this approximation is valid in the good solvent region where the intermolecular penetration is not significant. We observed that it does not agree with the data below $\bar{S} R_{H}<0.6$. It is interesting to note that the hard sphere calculations by Altenberger and Deutch ${ }^{13}$ (AD), which yield $k_{D}=2$, coincide exactly with $(\mathrm{AB})$, and closely with (Y) at $S R_{H}=1$. Since the experimental results in the good solvents happen to be in the vicinity of $\bar{S} R_{H}=1$, and agree reasonably well with (AD). Adam and Delsanti ${ }^{1+}$ concluded that the hard sphere model is adequate to interpret the data in good solvents. (They found $\varphi \equiv{ }_{D_{0}}^{S} \sim M^{0.8}$. or $k_{D}$ is molecular weight independent for polystyrene in benzene. We have not included their results in Figure?

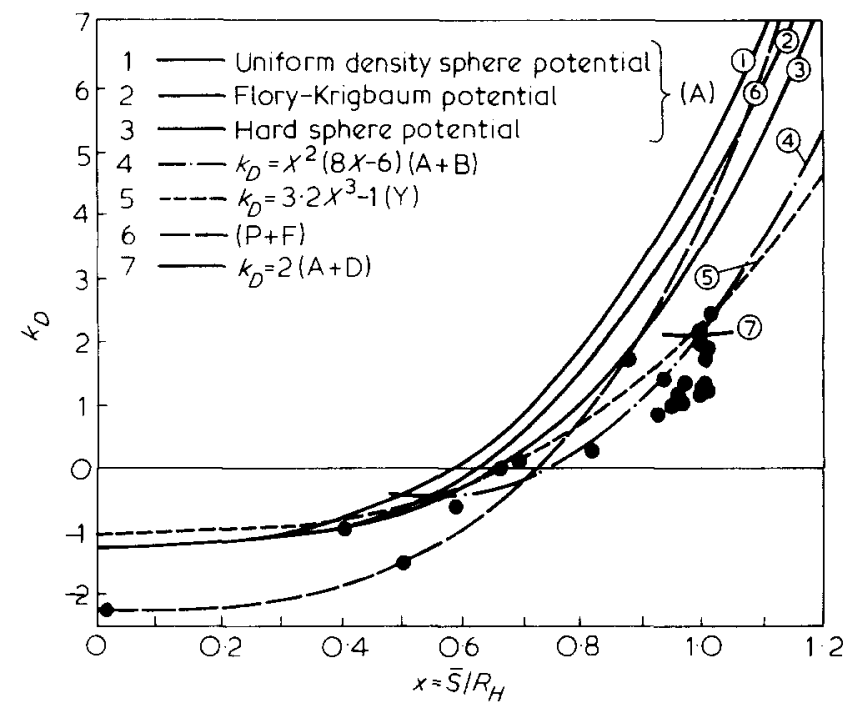

Figure 2 The variation of $k_{D}$ as a function of $\bar{S} / R_{H}$, together with theoretical preductions of various models

because the values of $A_{2}$ were not reported in their experiment). Figure 2 shows that the hard sphere model does not predict the variation of $k_{D}$ with temperature when the solvent becomes poor.

The calculations by Pyun and Fixman ${ }^{\natural}$ (PY). which yield $k_{D}=8\left(\bar{S} R_{H}\right)^{3}-7.1+\kappa(A)$ where $h($ A) takes into account the diffusion of overlapping polymers using prolate rigid ellipsoid model, seem to predict a faster rise with $\bar{S} R_{H}$ than the data indicate in the good solvent limit. Similar trends are followed by the recent calculations by Akcasu $^{11}$ (A) using three different models for the intermolecular interaction.

It is interesting to observe that all the theoretical models predict $k_{D}=0$ somewhere in the vicinity of $R_{H}$ $=0.72$. implying that the diffusion coefficient becomes 
independent of concentration at temperatures and molecular weights corresponding to $\bar{S} / R_{H} \cong 0.72$. This value of $\bar{S} / R_{H}$ separates the good solvent behaviour where the diffusion coefficient increases with concentration, from the $\theta$-solvent behaviour where it decreases with concentration.

As discussed in the accompanying paper ${ }^{11}$, the value of $k_{D}$ at the theta temperature, which essentially determines the behaviour of $k_{D}$ in the entire $\theta$-solvent region, is sensitive to the models used to describe the translational diffusion of an overlapping pair of molecules. The value $k_{D}$ $=-2.21$ at $\bar{S} / R_{H}=0$ predicted by (PF) seems to agree best with the experimental value $\mathrm{e}^{2.3}$. However, in slightly better solvents the predictions by (Y) and (A) seem to agree somewhat better with the data. In view of large uncertainties in the experimental values, it is not possible to draw more definite conclusions about the validity of the various theoretical models.

The calculations by $(\mathrm{AB})$ and $(\mathrm{A})$ are based on the first cumulant $\Omega(q)^{15}$. At zero concentration, $\Omega(q) / q^{2}$ yields ${ }^{16}$, as $q \rightarrow 0$, the short-time diffusion-coefficient $D_{0}$ of a polymer molecule as calculated originally by Kirkwood ${ }^{17} . D_{0}$ does not take into account the coupling between the translational and internal motions of the polymer. This coupling, which arises from the hydrodynamic interaction between monomers, gives rise to a small correction in the long time limit, as pointed out by Horta and Fixman ${ }^{18}$ in 1968 , and again by Fixman ${ }^{19}$ recently. The long time or steady-state diffusion coefficient then becomes $D=D_{0}-D_{1}$. Since the correction term $D_{1}$ is estimated to be no more than $1.67 \%$ for flexible chains ${ }^{19}$, which is well within the error of the measurement, we have not differentiated between $D$ and $D_{0}$ in the interpretation of the data through $\Omega(q)$.

In conclusion, more precise data is needed on the concentration dependence of the diffusion coefficient as a function of temperature and molecular weight on the theoretical side, more precise theoretical information is needed as regard to the diffusion coefficient of an isolated pair of molecules, that determines ${ }^{11}$ the concentration dependence of the diffusion coefficient near the theta temperature. Computer calculations similar to those recently published by Olaj, et $a .^{20}$, in which the interaction of a pair of chains is investigated, may provide more quantitative information in this respect; thereby eliminating the model dependence of the theoretical predictions in theta region.

\section{ACKNOWLEDGEMENTS}

We gratefully acknowledge the stimulating discussions with Drs I. C. Sanchez, E. A. DiMarzio, C. M. Guttman and F. L. McCrackin at the National Bureau of Standards. Acknowledgement is also made to donors of the Petroleum Research Fund, administered by the American Chemical Society, for partial support of A. Z. Akcasu.

\section{REFERENCES}

1 Yamahawa, H., 'Modern Theory of Polymer Solutions', Harper and Row, New York, (1971)

Han, C. C. Polymer 1979, 20, 259

3 Gulari, E.. Gulari, E., Tsunashima, Y. and Chu, B. Polymer 1979 20, 347

Yamakawa, H. J. Chem. Phys. 1962, 36, 2995

Imai, S. J. Chem. Phys. 1969, 50, 2116

Pyun, C. W. and Fixman, M. J. Chem. Phys, 1964, 41, 937

Han, C. C. Rev. Sci. Instrum. 1978, 49, 31

For example, see B. Chu, 'Laser Light Scattering', Academic Press, New York, (1974)

Mandema, W. and Zeldenrust, H. Polymer 1977, 18, 835

Selser, J. Macromolecules 1981, 14, 346

Akcasu, A. Z. Polymer 1981, 22, 1169

Akcasu, A. Z. and Benmouna, M. Macromolecules 1978, 11, 1193

Altemberger, A. R. and Deutch, J. M. J. Chem. Phys. 1973, 59, 894 Adam, M. and Delsanti, M. Macromolecules 1977, 10, 1229

Akcasu, A. Z. and Gürol, H. J. Polym. Sci., Polym. Phys. Edn. 1976, 14, 1

Akcasu, A. Z., Benmouna, H. and Han, C. C. Polymer 1980, 21, 866 Kirkwood, J. G. J. Polym. Sci. 1954, 12,1

Horta, A. and Fixman, M. J. Am. Chem. Soc. 1968, 90, 3048 Fixman, M., to be published (private communication)

Olaj, D.F., Lantschbauer, W. and Pelinka, K. H. Macromolecules 1980, 13, 299

Berry, G. C. J. Chem. Phys. 1966, 44, 4550

22 McIntyre, D., Wims, A., Williams, L. C. and Mandelkern, L. J. Chem. Phys. 1962, 66, 1932 